Check for updates

Cite this: RSC Adv., 2018, 8, 10277

Received 13th December 2017

Accepted 5th March 2018

DOI: 10.1039/c7ra13316b

rsc.li/rsc-advances

\section{Determination of six organophosphorus pesticides in water samples by three-dimensional graphene aerogel-based solid-phase extraction combined with gas chromatography/mass spectrometry}

\begin{abstract}
P. Sun, ${ }^{a b}$ Y. L. Gao, ${ }^{b}$ C. Xu ${ }^{b}$ and Y. F. Lian (D)*a
In the present study, a three-dimensional graphene aerogel (3D-GA), synthesised by chemical reduction of an aqueous solution of graphene oxides (GOs) with ethylenediaminethermal by a simple water bath method followed by freeze-drying treatment, was used for the solid-phase extraction (SPE) of six organophosphorus pesticides (OPPs) (i.e. trichlorfon, dimethoate, ethoprophos, parathion, fenitrothion and fenthion) from water samples. The target analytes were extracted using packed SPE cartridges and then eluted with tetrahydrofuran. The eluate was collected and dried with high-purity nitrogen gas at room temperature. After redissolving in acetone, the residue was analysed using gas chromatography/ mass spectrometry (GC/MS). The proposed method demonstrated a good linearity between 0.5 and 500 $\mu \mathrm{g} \mathrm{L}^{-1}$ with the correlation coefficient of $0.9990-0.9998$. The limits of detection (LODs) $(\mathrm{S} / \mathrm{N}=3)$ and the limits of quantification (LOQs) $(S / N=10)$ for the six OPPs pesticides were in the range of $0.12-0.58$ $\mu \mathrm{g} \mathrm{L}^{-1}$ and $0.41-1.96 \mu \mathrm{g} \mathrm{L^{-1 }}$, respectively. The accuracy of the present method was evaluated by measuring the recovery of the spiked samples, which ranged from $93.8 \%$ to $104.2 \%$ with relative standard deviations (RSDs) of $1.1-5.6 \%$. The established method was successfully applied to the determination of the target analytes in environmental water samples including tap water, river water, drinking water and lake water, demonstrating its great potential for the determination of OPPs in water.
\end{abstract}

\section{Introduction}

Because of their high efficiency and broad spectrum, organophosphorus pesticides (OPPs) are widely used for protecting crops against pests, thereby increasing the productivity of the harvest. However, the massive use of pesticides has already contributed to the current levels of environmental pollution especially in water systems. Therefore, the development of accurate and sensitive analytical methods for the simultaneous determination of trace levels of OPPs that facilitate the assessment of risk is in increasing demand.

Since the analytes of interest are present in environmental water samples at low concentrations, the selection of an adequate sample preparation technique is essential for achieving an accurate determination of OPPs in water. Liquidliquid extraction (LLE), ${ }^{1}$ solid-phase extraction (SPE), ${ }^{2}$ solidphase microextraction (SPME), ${ }^{3}$ dispersive liquid-liquid microextraction (DLLME) ${ }^{4}$ molecularly imprinted solid phase extraction(MISPE), ${ }^{5}$ liquid-phase microextraction (LPME), ${ }^{6}$

${ }^{a}$ Key Laboratory of Functional Inorganic Material Chemistry, Ministry of Education, School of Chemistry and Materials Science, Heilongjiang University, Harbin 150080, China. E-mail: chyflian@hlju.edu.cn

${ }^{b}$ Analytical Centre, Heilongjiang Bayi Agricultural University, Daqing163319, China microwave extraction, ${ }^{7}$ dispersive SPE (d-SPE), ${ }^{8}$ hollow fibre liquid-phase microextraction (HF-LPME) ${ }^{9}$ and single-drop microextraction (SDME) ${ }^{10}$ have been employed for the extraction of OPPs from water samples. Among them, the well-known SPE technique is presently the most extended method for the preconcentration of OPP pesticide residues from water samples mainly due to its large enrichment capacity.

In the SPE procedure, the adsorbent plays an important role in achieving enhanced analyte extraction efficiency, since the extraction takes place by the adsorption of the target compound on the surface of adsorbents. The choice of an appropriate adsorbent is therefore a critical factor in order to obtain a satisfactory recovery. Many materials, such as magnetic nanoparticles, ${ }^{11}$ carbon nanotubes,${ }^{12}$ graphitised carbon black ${ }^{13}$ macroporous resin ${ }^{14}$ and graphite, ${ }^{15}$ have already been used as adsorbents for the determination of pesticides from a variety of samples. In this context, one of the novel adsorbents that have been fabricated in order to enhance the performance of SPE techniques and satisfy the requirements of the highly sensitive and selective green analytical chemistry is threedimensional (3D) graphene. Formed by graphene sheets, 3D graphene retains the excellent physical and chemical properties of graphene, and its 3D structure gives rise to its many superior characteristics. Nowadays, 3D graphene is successfully used as 
an adsorbent for the preconcentration and removal of dyes, ${ }^{\mathbf{1 6 , 1 7}}$ heavy metals, ${ }^{18}$ organophosphorus pesticides, ${ }^{19}$ phenols ${ }^{20}$ and retardants. ${ }^{21}$

In this work, a 3D graphene aerogel (3D-GA) was synthesised by chemical reduction of an aqueous solution of graphene oxides (GOs) followed by freeze-drying treatment. Making full use of its porous and large surface area, such prepared 3D-GA was packed in an SPE cartridge, and its adsorption behaviour towards OPPS was investigated. The SPE parameters affecting the extraction efficiency were optimised, and the target analytes were quantified through gas chromatography/mass spectrometry (GC/MS). We consider that this method, which was utilised for the determination of OPPs in water samples, can provide some reference for the determination of other pesticide residues in water.

\section{Experimental}

\subsection{Reagents and materials}

Trichlorfon, ethoprophos, dimethoate, fenitrothion, parathion and fenthion with $99.5 \%$ purity were purchased from the AgroEnvironmental Protection Institute, Ministry of Agriculture, China. High-performance liquid chromatography (HPLC) grade methanol, ethanol, acetone, tetrahydrofuran and acetonitrile were purchased from Fisher Scientific, Massachusetts, USA. A microporous membrane of $0.22 \mu \mathrm{m}$ was obtained from Dikma Technologies (Beijing, China).

Tap water, river water, drinking water and lake water samples were collected randomly from Daqing, China. All water samples were filtered through a $0.22 \mu \mathrm{m}$ membrane syringe filter to remove the suspended solids and then stored at $4{ }^{\circ} \mathrm{C}$.

\subsection{Instruments}

Chromatographic analysis was performed on a Shimadzu GC/ MS QP2010 (Shimadzu Co., Japan) equipped with EI ion sources, and the system was controlled by the GC/MS solution Ver.2 software (Shimadzu Co., Japan). Scanning electron microscopy (SEM) micrographs were taken using a Hitachi S-4800 microscope. The samples were treated by nitrogen sweeping in a water bath system (Tianjin Automatic Science Instrument Co., Ltd.). FT-IR spectra were recorded on a 1600 series Perkin-Elmer (MA, USA) with a resolution of $4 \mathrm{~cm}^{-1}$. Powder X-ray diffraction (XRD) was carried out on a Bruker D8 X-ray diffractometer using $\mathrm{Cu} \mathrm{K} \alpha$ radiation. SPE was carried out on an Extrapid SPE apparatus from Beijing Labtech Instruments Co., Ltd.

\subsection{Preparation of 3D-GA}

Graphite oxide was synthesised by a modification of Hummers method as follows. ${ }^{22,23}$ A $100 \mathrm{~mL}$ beaker containing $1 \mathrm{~g}$ of graphite powder and $23 \mathrm{~mL}$ of concentrated $\mathrm{H}_{2} \mathrm{SO}_{4}$ was placed into an ice-colded water bath pot, and followed by stirring. When the graphite powder was well dispersed, $3.0 \mathrm{~g}$ of $\mathrm{KMnO}_{4}$ was added under stirring and the mixture was stirred and kept at $45{ }^{\circ} \mathrm{C}$ for $1.5 \mathrm{~h}$. After $120 \mathrm{~mL}$ deionized water was slowly added, the beaker was maintained $90{ }^{\circ} \mathrm{C}$ for $10 \mathrm{~min}$, and then $20 \mathrm{~mL}$ of $\mathrm{H}_{2} \mathrm{O}_{2}(30 \%, \mathrm{v} / \mathrm{v})$ were added, causing yellow mixture along with bubbling. When the bubbling stopped, the suspension was cooled down and centrifuged for 5 min at $10000 \mathrm{rpm}$, and then the centrifugal deposit was washed with $10 \% \mathrm{HCl}$ solution to remove metal ions followed with water until the $\mathrm{pH}$ became 5.0-6.0. After drying at $50{ }^{\circ} \mathrm{C}$, graphite oxide was obtained as grey brown powder.

The as-prepared graphite oxide $(0.4 \mathrm{~g})$ was dispersed in $100 \mathrm{~mL}$ of water and ultrasonicated for $1 \mathrm{~h}$. The resulting solution $(5 \mathrm{~mL})$ was placed in a test tube with an i.d. of $12 \mathrm{~mm}$, and its $\mathrm{pH}$ was adjusted to weakly alkaline with ammonia. Ethylenediamine ( $20 \mu \mathrm{L}$ of a $50 \mathrm{~mol} \mathrm{~L}^{-1}$ aqueous solution) was added to the dispersion. After well mixed, the test tube was kept in a water bath pot and maintained $80^{\circ} \mathrm{C}$ for $24 \mathrm{~h}$ to prepare the graphene hydrogel. Finally, the black cylinder-like graphene hydrogel was washed with ethanol solution $(20 \%, v / v)$ for $6 \mathrm{~h}$ to remove the residual soluble impurities and to prevent it from shrinkage and collapse during the followed freeze-drying. After vacuum freeze-drying for $48 \mathrm{~h}$, the 3D-GA was formed.

\subsection{SPE procedures}

The 3D-GA was placed between an upper frit and a lower frit in an empty $3 \mathrm{~mL}$ SPE cartridge with an i.d. of $8.9 \mathrm{~mm}$, and then properly compacted to retain its super surface area and porousness for the adsorption OPPs. Prior to extraction, the SPE cartridge was preconditioned with $3 \mathrm{~mL}$ of methanol, $3 \mathrm{~mL}$ of acetone, $3 \mathrm{~mL}$ of acetonitrile, $3 \mathrm{~mL}$ of tetrahydrofuran and $10 \mathrm{~mL}$ of double-distilled water. $40 \mathrm{~mL}$ of water sample was passed through the cartridge at a flow rate of $1 \mathrm{~mL} \mathrm{~min}^{-1}$ on an Extrapid SPE apparatus, and then $5 \mathrm{~mL}$ of tetrahydrofuran was used to elute the analytes retained on the 3D-GA adsorbent. The eluent was collected and dried by nitrogen at room temperature, and $1 \mathrm{~mL}$ of acetone was used to redissolve the residue. After filtration through a $0.22 \mu \mathrm{m}$ membrane, the acetone solution was subjected to GC/MS analysis.

\subsection{GC/MS analysis}

Chromatographic separation was performed on an Rtx-50 (Shimadzu Co., Japan) capillary column $(30 \mathrm{~m} \times 0.25 \mathrm{~mm}$ i.d., 0.25 $\mu \mathrm{m}$ film thickness) under the following instrumental conditions: helium (99.999\%) was used as the carrier gas; the total flow was $26.5 \mathrm{~mL} \mathrm{~min}^{-1}$; the column flow was $1.2 \mathrm{~mL} \mathrm{~min}^{-1}$ and the injector temperature was $220{ }^{\circ} \mathrm{C}$. The injection mode was splitless for $1 \mu \mathrm{L}$ samples, and the oven temperature was programmed as follows: the initial temperature was $70{ }^{\circ} \mathrm{C}$ (held for 2 min) and successively increased to $160^{\circ} \mathrm{C}$ at a rate of $15^{\circ} \mathrm{C} \mathrm{min}{ }^{-1}$ (held for $2 \mathrm{~min}$ ), to $210^{\circ} \mathrm{C}$ at a ramp rate of $10^{\circ} \mathrm{C} \mathrm{min}^{-1}$ (held for $2 \mathrm{~min}$ ), to $220^{\circ} \mathrm{C}$ at a ramp rate of $5^{\circ} \mathrm{C} \mathrm{min}^{-1}$ (held for $2 \mathrm{~min}$ ) and to $270{ }^{\circ} \mathrm{C}$ at a ramp rate of $10{ }^{\circ} \mathrm{C} \mathrm{min}^{-1}$ (held for $14 \mathrm{~min}$ ). MS was performed in the EI ionisation mode with an energy of ionisation, an ionisation temperature and an interface temperature of $70 \mathrm{eV}, 260{ }^{\circ} \mathrm{C}$ and $270{ }^{\circ} \mathrm{C}$, respectively.

\section{Results and discussion}

\subsection{Characterisation of the 3D-GA}

The characterisation of the as-prepared 3D-GA was carried out using SEM, XRD and a Fourier transform infrared (FTIR) 
spectrometer. The SEM image shown in Fig. 1 indicates that the 3D-GA is consisted of a honeycomb 3D porous networks with an average pore size of about 100 micrometres, and the pore walls are constructed from partially overlapped thin graphene sheets. Such kinds of 3D porous networks are conducive to the migration and adsorption of OPPs molecules in/on the 3D-GA, thus providing a favourable platform for the complete extraction of OPP residues from environmental water.

The FTIR spectrum shown in Fig. 2 exhibits absorption bands at $3394 \mathrm{~cm}^{-1}$ that are attributable to $\mathrm{O}-\mathrm{H}$ stretching vibration. A distinct band at $1734 \mathrm{~cm}^{-1}$ confirms the presence of carbonyl $\mathrm{C}=\mathrm{O}$ groups that were not fully removed after chemical reduction. The band at $1618 \mathrm{~cm}^{-1}$ can be assigned to the stretching vibrations of $\mathrm{C}=\mathrm{C}$ conjugated bonds. In turn, the bands at 1228 and $1049 \mathrm{~cm}^{-1}$ can be attributed to the epoxy C-O-C stretching vibration, which suggests the presence of epoxide bonds ${ }^{24}$ that still remained in the 3D-GA.

Shown in Fig. 3 are the XRD patterns of graphene oxide (GO) and the 3D-GA. It is obvious that GO has a larger interlayer distance than graphite according to the corresponding diffraction peak $\left(2 \theta=11.5^{\circ}\right) .{ }^{25}$ However, this diffraction peak is completely disappeared after chemical reduction and a broad one centred at $24.6^{\circ}$ is turned up, corresponding to the interlayer distance in 3D-GA. Therefore, it is concluded that graphene oxide is largely reduced to thin graphene sheets by ethylenediamine.

\subsection{Optimisation of SPE procedures}

Several factors affecting the SPE performance were investigated in this paper. These parameters included the type and volume of the elution solvent, the $\mathrm{pH}$ value of water sample and the volume of water sample for a definite amount of 3D-GA.

3.2.1 Optimisation of the elution solvent. In the SPE methods, selection of the type of elution solvent is of vital importance for the extraction efficiency of the analytes. It is well-known that graphitic substrates adsorb compounds based on hydrophobic interactions. However, graphite oxide were not completely reduced during the graphene aerogel preparation. Thus, some graphene oxides are co-existing with graphene, i.e. there are some hydrophilic chemical groups on the surface of graphene, which plays a role to adjust the hydrophobicity of $3 \mathrm{D}$ -

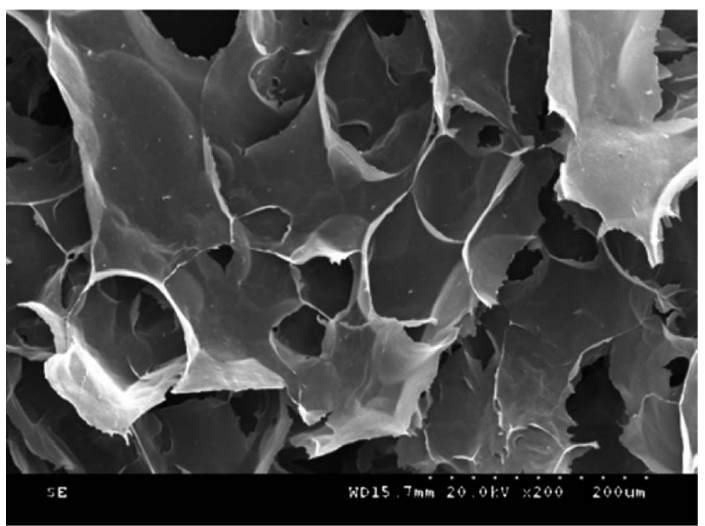

Fig. 1 SEM image of 3D-GA.

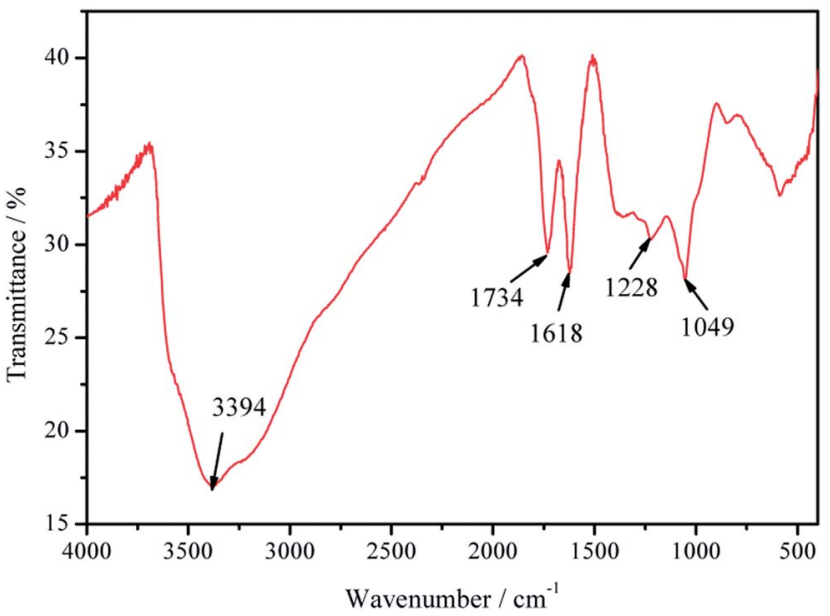

Fig. 2 FTIR spectrum of the 3D-GA.

GA. Thus, 3D-GA could be wetted by water and used as adsorbent for OPPs. Because of the large hydrophobic interaction between 3D-GA and OPPs, the OPPs could be completely adsorbed on 3DGA from water. On the other hand, methanol, acetone, acetonitrile and tetrahydrofuran were used as eluent to elute OPPs from
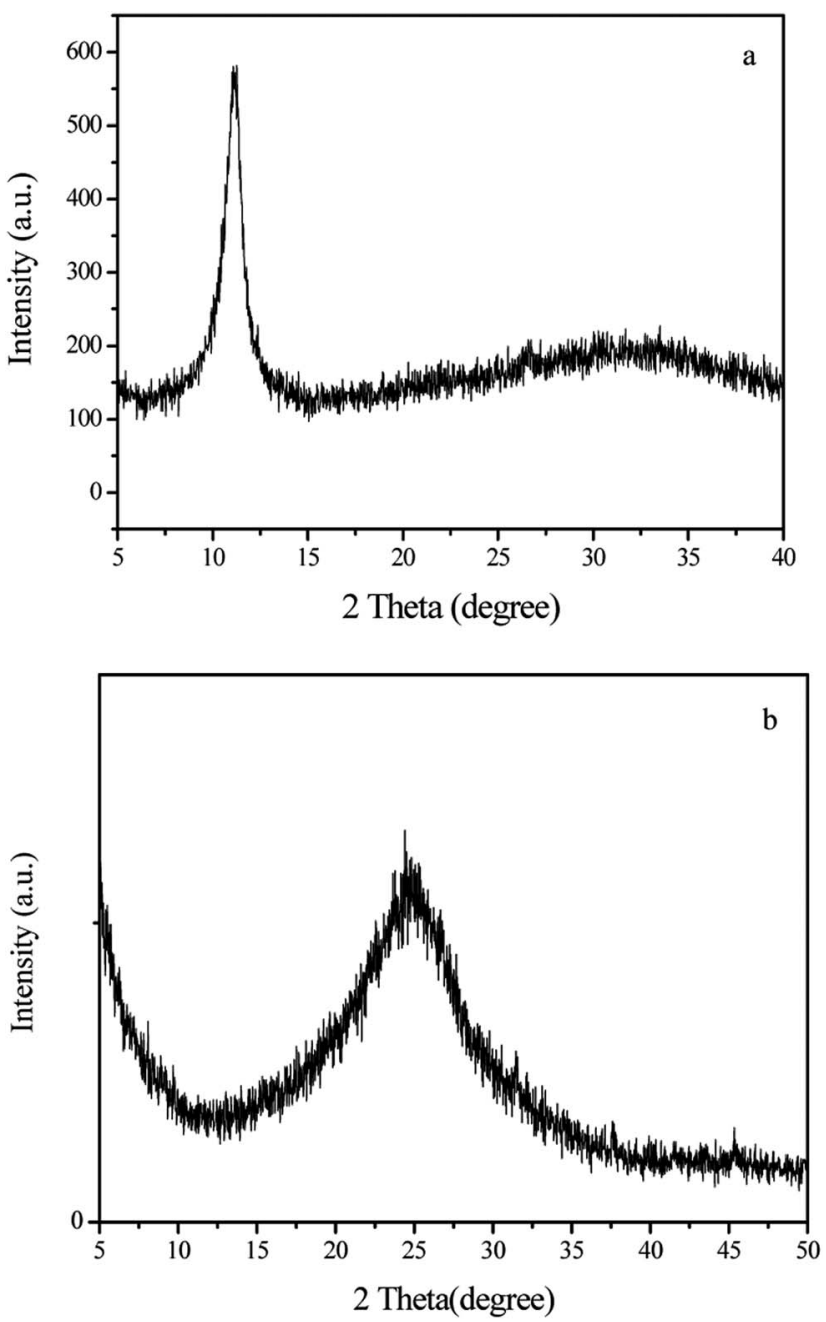

Fig. 3 XRD patterns of graphene oxide (a) and 3D-GA (b). 


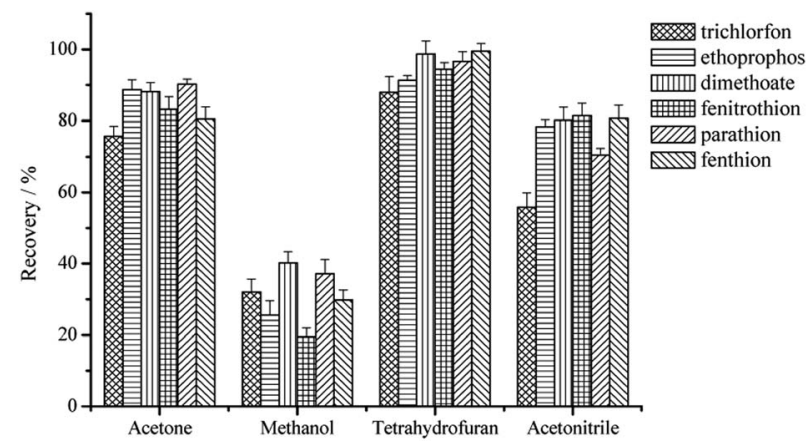

Fig. 4 The effect of the type of eluent on the extraction recoveries of the six OPPs.

3D-GA adsorbent. It can be seen from Fig. 4 that tetrahydrofuran with the highest recovery of OPPs is the best elution solvent. Here shows the large effect of the polarity of eluent on the performance of elution. Largely because of the competitive hydrophobic interaction between elution solvent and OPPs to that between 3DGA and OPPs, the more polar the elution solvent is, the worst the elution performs. Since the polarity of tetrahydrofuran is the smallest among the applied elution solvents, tetrahydrofuran is chosen as the best eluent for the OPPs adsorbed on 3D-GA.

3.2.2 Optimisation of the elution solvent volume. The elution solvent volume was evaluated in the range of 1-10 mL. As shown in Fig. 5, the highest recovery of target compounds was obtained with increasing tetrahydrofuran volumes. The extraction recoveries for all the six analytes increased with the increase in the volume of tetrahydrofuran, reaching the maximum value with $5 \mathrm{~mL}$ of tetrahydrofuran. Upon further increase in the volume of tetrahydrofuran, the extraction recoveries kept almost constant. This means that $5 \mathrm{~mL}$ of tetrahydrofuran is enough to eluate completely the target compounds adsorbed on a definite amount of 3D-GA. Therefore, the elution solvent volume adopted for SPE in this study was $5 \mathrm{~mL}$.

3.2.3 Optimisation of sample $\mathbf{p H}$. The $\mathrm{pH}$ value of a solution sample has great effects both on the chemical forms of target compound and on the adsorption between target

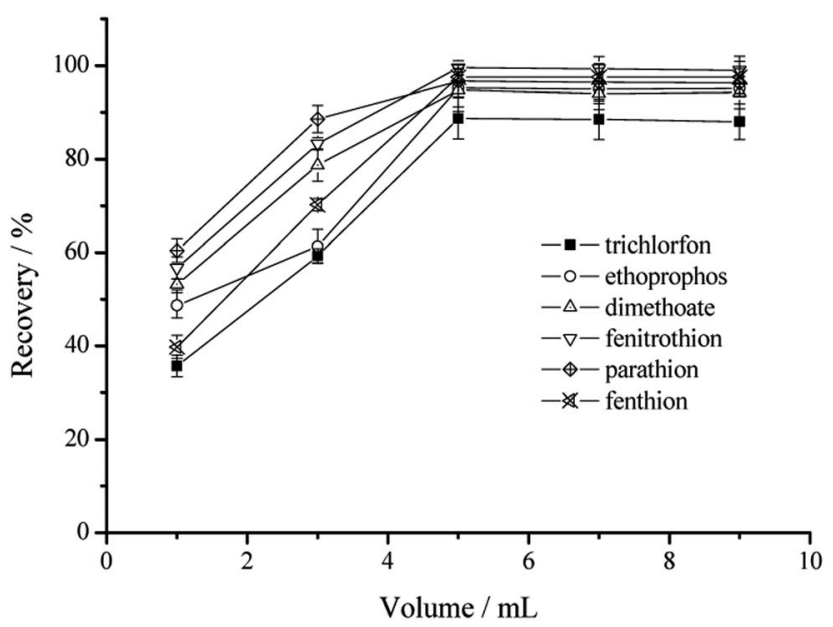

Fig. 5 The effect of the volume of the eluent on the extraction recoveries of the six OPPs.

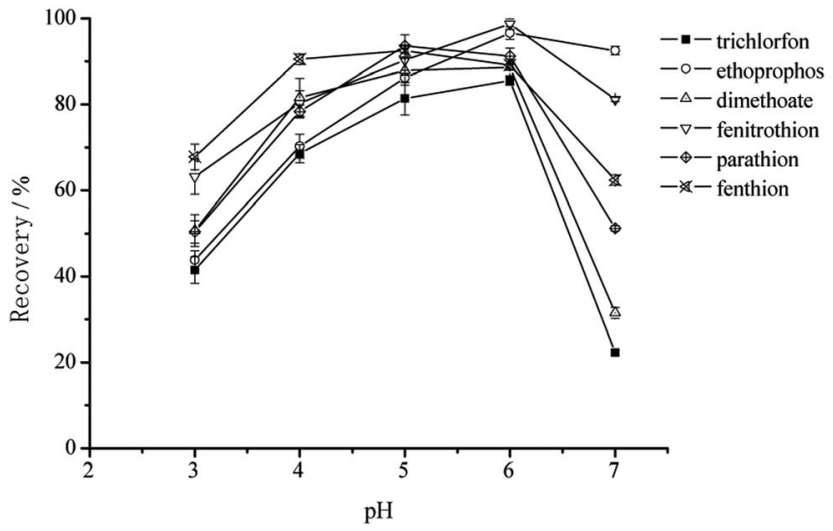

Fig. 6 The effect of sample $\mathrm{pH}$ on the extraction recoveries of the six OPPs.

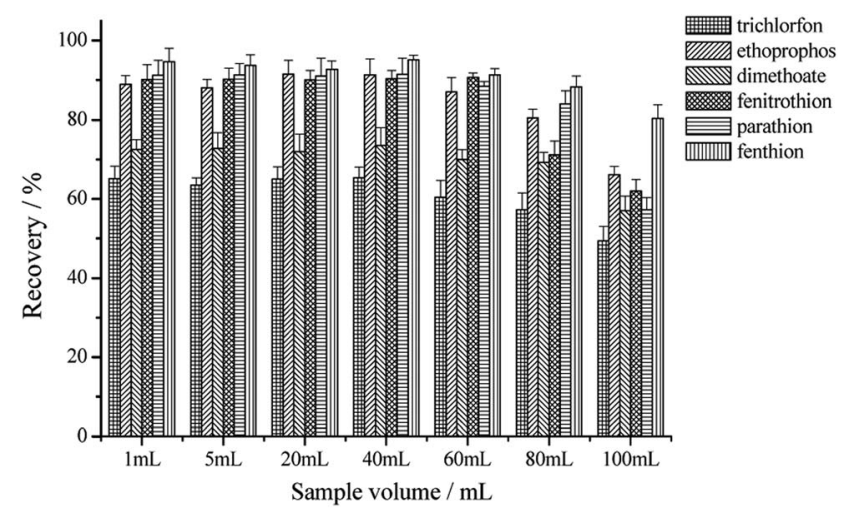

Fig. 7 The effect of the sample volume on the recoveries of the six OPPs.

compound and adsorbent. Thus, the $\mathrm{pH}$ of the sample solution should be adjusted in order to ensure that the target compound is efficiently adsorbed. Since the analytes are relatively stable at $\mathrm{pH}$ 4.0-6.0, the $\mathrm{pH}$ value of mixed standard solution was adjusted to 3.0-7.0 with acetate for the SPE procedures.

As shown in Fig. 6, the recovery is the highest at $\mathrm{pH}$ value of 6.0. Since OPPs are relatively unstable at $\mathrm{pH}>7$, some of them may be decomposed, leading to a low recovery of OPPs. On the other hand, the surface of graphene sheets is negatively charged. When the $\mathrm{pH}$ value is changed to $3-5, \mathrm{H}^{+}$would compete with OPPs to adsorb on 3D-GA, also leading to a low recovery of OPPs.

Table 1 Linearity, LOD and LOQ for the six OPPs in stand water samples $(n=6)$

\begin{tabular}{llllll}
\hline Analyte & $\begin{array}{l}\text { Linearity range } \\
\left(\mu \mathrm{g} \mathrm{L}^{-1}\right)\end{array}$ & $r$ & $\begin{array}{l}\text { LOD } \\
\left(\mu \mathrm{g} \mathrm{L}^{-1}\right)\end{array}$ & $\begin{array}{l}\text { LOQ } \\
\left(\mu \mathrm{g} \mathrm{L}^{-1}\right)\end{array}$ & RSD \% \\
\hline Trichlorfon & $2 \sim 500$ & 0.9990 & 0.58 & 1.96 & 4.6 \\
Ethoprophos & $0.5 \sim 500$ & 0.9994 & 0.18 & 0.62 & 1.2 \\
Dimethoate & $1 \sim 500$ & 0.9993 & 0.32 & 1.06 & 3.4 \\
Fenitrothion & $1 \sim 500$ & 0.9992 & 0.21 & 0.68 & 2.8 \\
Parathion & $0.5 \sim 500$ & 0.9998 & 0.26 & 0.88 & 1.2 \\
Fenthion & $1 \sim 500$ & 0.9995 & 0.12 & 0.41 & 4.2
\end{tabular}


Table 2 Recoveries (RSDs) of OPPs from spiked water samples $(n=6)$

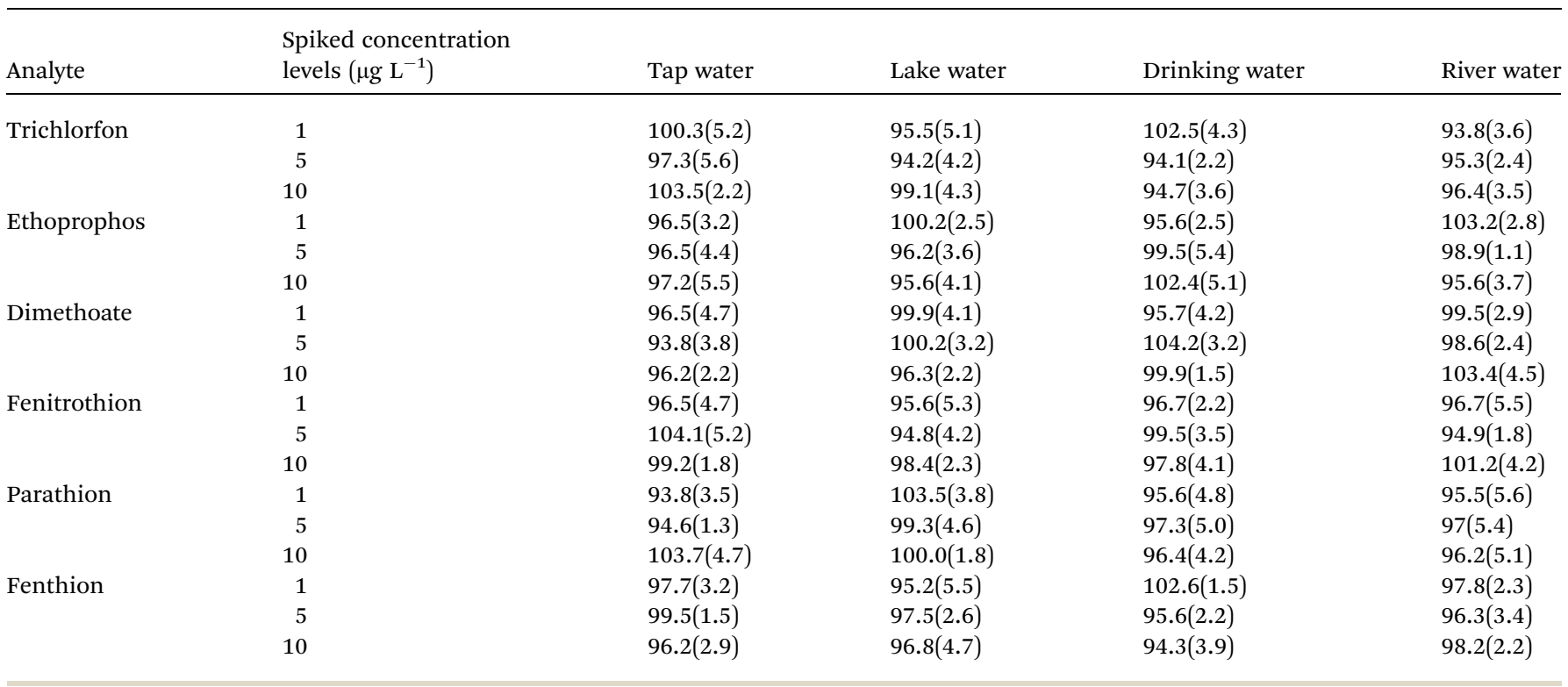

3.2.4 Optimisation of the sample volume. Based on the same amounts of 3D-GA adsorbents, the stand water sample containing six OPPs with each concentration of $100 \mu \mathrm{g} \mathrm{L}^{-1}$ was applied to the optimization of sample volume. According to "the environmental quality standards for surface water (GB 38382002)", the maximum residue limit (MRL) of OPPs is 2 to $50 \mu \mathrm{g}$ $\mathrm{L}^{-1}$. Moreover, in line with water industry standard "water quality-determination of organophosphorus pesticides-solid phase extraction/gas chromatography (SL 739-2016)", calibration curves are made in the concentration range from $10 \mu \mathrm{g} \mathrm{L}^{-1}$ to $200 \mu \mathrm{g} \mathrm{L}^{-1}$. In order to ensure the universal applicability of this proposed method, a concentration of $100 \mu \mathrm{g} \mathrm{L}{ }^{-1}$ for each OPP was chosen for the optimization of sample volume.

The packed SPE cartridge was loaded with $20-120 \mathrm{~mL}$ of mixed OPPs standard solutions at an overall concentration of $600 \mu \mathrm{g} \mathrm{L}^{-1}$ (100 $\mu \mathrm{g} \mathrm{L}^{-1}$ for each compound). The rest of the experimental conditions were set as described in Section 2.4. It could be seen from Fig. 7 that the extraction recoveries do not change significantly for the six OPPs when the water sample volume increases from 1 to $40 \mathrm{~mL}$. This means that for a definite amount of 3D-GA the adsorbed OPPs tend to be saturated and the enrichment factor is gradually increased with the increase in water sample volume. However, the extraction recoveries are significantly decreased for the six OPPs when the water sample volume is larger than $60 \mathrm{~mL}$. This means that OPPs in $60 \mathrm{~mL}$ water sample are supersaturated for the same amount of 3D-GA and then some OPPs can not be recovered. Therefore, the loading volume of water sample has been identified as $40 \mathrm{~mL}$.

\subsection{Application of packed SPE in water samples}

3.3.1 Linearity, repeatability and limits of detection (LODs). $40 \mathrm{~mL}$ of water samples containing six concentration levels of OPP standards were SPE extracted and analysed under the optimised experimental conditions. Shown in Table 1 are
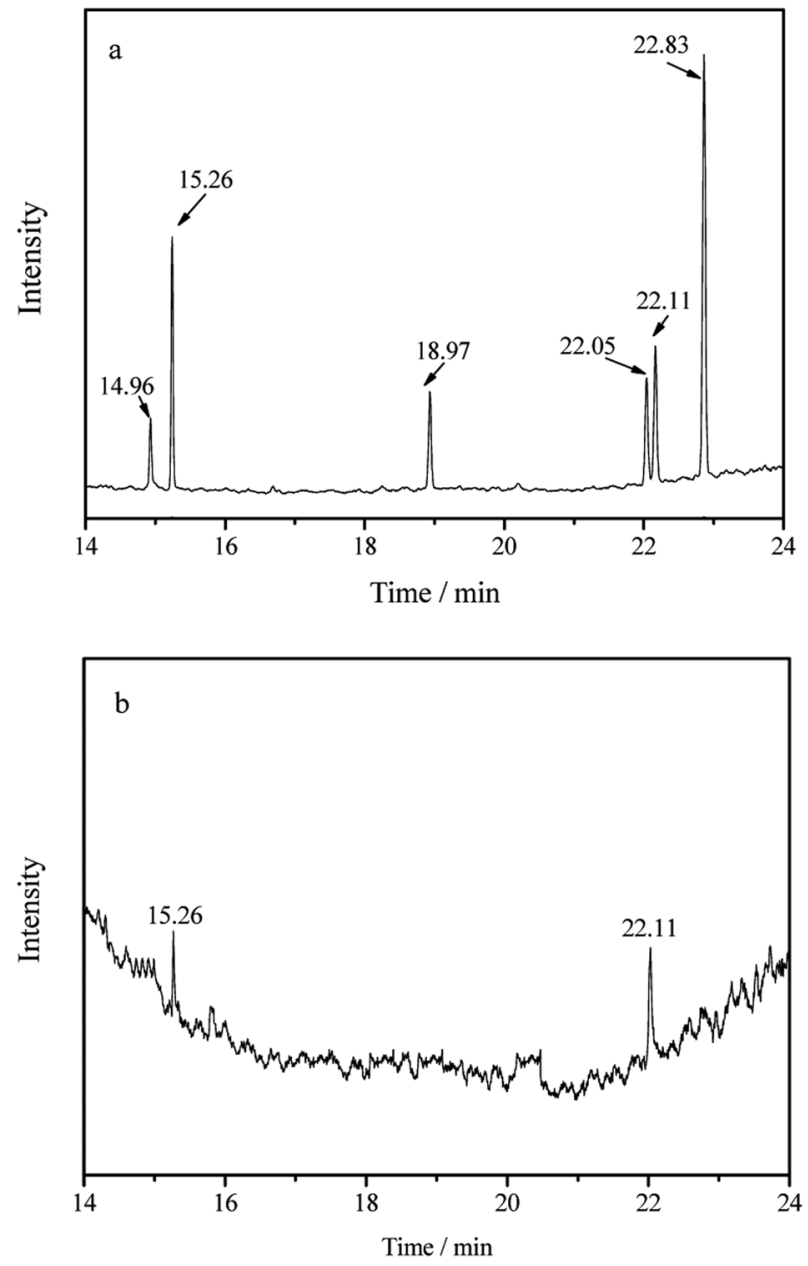

Fig. 8 TIC chromatograms of the spiked water sample (a) and a real river water sample (b). Trichlorfon $\left(t_{R}=14.96 \mathrm{~min}\right)$, ethoprophos $\left(t_{R}=\right.$ $15.26 \mathrm{~min})$, dimethoate $\left(t_{R}=18.97 \mathrm{~min}\right)$, fenitrothion $\left(t_{R}=22.05 \mathrm{~min}\right)$, parathion $\left(t_{R}=22.11 \mathrm{~min}\right)$ and fenthion $\left(t_{R}=22.83 \mathrm{~min}\right)$. 
Table 3 Analytical results for the determination of the six OPPs in environmental water samples

\begin{tabular}{lllll}
\hline Analyte & Tap water & Lake water & Drinking water & River water \\
\hline Trichlorfon & N.D. & N.D. & N.D. & N.D. \\
Ethoprophos & N.D. & N.D. & N.D. & 0.54 \\
Dimethoate & N.D. & N.D. & N.D. & N.D. \\
Fenitrothion & N.D. & N.D. & N.D. & N.D. \\
Parathion & N.D. & N.D. & N.D. & 0.76 \\
Fenthion & N.D. & N.D. & N.D. & N.D.
\end{tabular}

the linearity, LOD and LOQ for the six OPPs in stand water samples. A good linear relationship between the mass spectroscopic peak area $(A)$ and the OPP concentration in spiked water samples $\left(C, \mu \mathrm{g} \mathrm{L}^{-1}\right)$ was obtained in the range of $0.5-500 \mu \mathrm{g} \mathrm{L}^{-1}$ with the correlation coefficient being 0.9990-0.9998. The LOD $(\mathrm{S} / \mathrm{N}=3)$ was calculated to be $0.12-0.58 \mu \mathrm{g} \mathrm{L}^{-1}$, and the LOQ (S/ $\mathrm{N}=10$ ) to be $0.41-1.96 \mu \mathrm{g} \mathrm{L}^{-1}$ for the six target compounds. The OPP standard solution was sampled continuously six times at concentrations of $100 \mu \mathrm{g} \mathrm{L^{-1 }}$, and the peak areas were recorded by GC/MS according to the precision of the instrument. The relative standard deviation (RSD) ranges were $1.2-4.6 \%$, indicative of the good precision of the present method. Therefore, the sensitivity of this method can be expected to meet the requirements of quantitative and qualitative analyses of real water samples.

In order to estimate the quantitative accuracy of the proposed method, OPP-free real water samples were analysed with spiked concentration levels of 1,5 and $10 \mu \mathrm{g} \mathrm{L}^{-1}$, respectively. Under the optimised conditions established above, the recoveries and RSDs of the six target compounds were calculated and listed in Table 2. It can be seen that the recoveries range from $93.8 \%$ to $104.2 \%$ and the RSD ranges from $1.1 \%$ to $5.6 \%$, revealing the excellent sensitivity of the proposed method.

3.3.2 Real water samples analysis. In order to investigate the performance of the present method in the determination of OPPs in water samples, spiked and real water samples were analysed with the proposed procedure. Water samples including tap water, river water, drinking water and lake water were collected from Daqing, China. After the above mentioned pretreatment, the samples were subjected to GC/MS analysis under the optimised conditions. Shown in Fig. 8 are the total ion current (TIC) chromatograms of the spiked sample and a real river water sample. It was obvious that ethoprophos and parathion were successfully detected in river water. The experimental results are summarised in Table 3. Trichlorfon, dimethoate, fenitrothion and fenthion were not found in all of the real water samples. Whereas, ethoprophos and parathion were detected in river water at concentrations lower than their LOQs, although they were not found in tap water, drinking water or lake water samples.

The real water and spiked water samples were simultaneous analyzed by the proposed procedure, and the analytical results from the spiked water samples confirmed that the proposed methodology could be applicable to the determination of trace OPPs in various water samples. On the other hand, OPPs are easily accumulated in river water, since they are often used as pesticides for agricultural purposes. Whereas, tap water, lake water and drinking water are rarely contaminated by OPPs, and then they were not detected.

\subsection{Comparison of developed method with others}

In order to evaluate the performance of the present method, a comparative study with other previously reported analytical technologies for the determination of OPPs including magneticSPE GC (MSPE-GC), ${ }^{2}$ microwave-assisted extraction and microSPE (MAE- $\mu$-SPE) GC/MS (MAE- $\mu$-SPE-GC/MS), ${ }^{3}$ LLME-HPLC$\mathrm{UV}^{6}{ }^{6}$ d-SPE-GC/MS ${ }^{8}$ elevated temperature-DLLME-GC (ETDLLME-GC), ${ }^{26}$ LLE-GC, ${ }^{27}$ and QuEChERS-GC-QTOF-MS ${ }^{28}$ was carried out. Listed in Table 4 are the experimental results including RSD, linearity range, LOD and recovery. It can be seen from Table 4 that the proposed method exhibits the best correlation coefficient, the highest recovery and the highest precision among the technologies listed. Moreover, the proposed method shows a limit of detection comparable to that of LLME-HPLC-UV, which is much lower than that of ETDMLLE-GC, d-SPE-GC/MS, LLE-GC, MAE- $\mu$-SPE-GC/MS, MSPEGC and QuEChERS-GC-QTOF-MS. Additionally, the proposed method also demonstrates relatively wider linearity range than that of LLME-HPLC-UV or MAE- $\mu$-SPE GC/MS, and covers lower linearity range than that of ET-DMLLE-GC, LLE-GC, MSPE-GC and QuEChERSGC-QTOF-MS. In summary, the identification and quantification of OPPs in water evidences that the advantages of the proposed method lie in low detection limit, high recovery, offering a novel and applicable procedure for the analysis of trace OPPs in water sample.

Table 4 Comparative study of the present method with others previously reported

\begin{tabular}{|c|c|c|c|c|c|c|c|}
\hline Method & Matrix & $r$ & $\begin{array}{l}\text { Linearity } \\
\text { range }\end{array}$ & $\begin{array}{l}\text { Recovery } \\
(\%)\end{array}$ & $\begin{array}{l}\text { Precision } \\
\text { (RSD \%) }\end{array}$ & LOD & Ref. \\
\hline LLME-HPLC-UV & Water and juice & 0.9984 & $0.5-400 \mu \mathrm{g} \mathrm{L^{-1 }}$ & $92.2-111.5$ & $<5.7$ & $0.10-0.35 \mu \mathrm{g} \mathrm{L}{ }^{-1}$ & 2 \\
\hline DMLL-GC & Aqueous samples & 0.998 & $2.6-1000 \mu \mathrm{g} \mathrm{L}^{-1}$ & $64-83$ & $<5.7$ & $0.82-1.65 \mu \mathrm{g} \mathrm{L}^{-1}$ & 3 \\
\hline d-SPE-GC/MS & Peanut oil & 0.9982 & $0.25-1000 \mu \mathrm{g} \mathrm{kg}^{-1}$ & $85.9-114.3$ & $<8.5$ & $0.7-1.6 \mu \mathrm{g} \mathrm{kg}^{-1}$ & 6 \\
\hline LLE-GC & Dried fruits & 0.9973 & $4.0-1000 \mu \mathrm{g} \mathrm{L}^{-1}$ & $70-110$ & $<7.3$ & $4-30 \mathrm{ng} \mathrm{mL}^{-1}$ & 8 \\
\hline MAE- $\mu-S P E-G C / M S$ & Vegetable and fruit & 0.9995 & $0.50-50 \mu \mathrm{g} \mathrm{kg}^{-1}$ & $93.5-104.6$ & $<8.7$ & $0.06-0.23 \mu \mathrm{g} \mathrm{kg}-1$ & 26 \\
\hline MSPE-GC & Water samples & 0.9990 & $100-1000 \mu \mathrm{g} \mathrm{L} \mathrm{L}^{-1}$ & $83-105$ & $<8.7$ & $<100 \mu \mathrm{g} \mathrm{L}^{-1}$ & 27 \\
\hline QuEChERS-GC-QTOF-MS & Fruits and vegetables & 0.9845 & $10-1000 \mu g \mathrm{~L}^{-1}$ & $70.0-115.9$ & $<19.5$ & $1.18-5.55 \mu \mathrm{g} \mathrm{kg}^{-1}$ & 28 \\
\hline 3D-GA-SPE-GC/MS & Water & 0.9998 & $0.5-500 \mu \mathrm{g} \mathrm{L}^{-1}$ & $93.8-104.2$ & $<5.6$ & $0.14-0.58 \mu \mathrm{g} \mathrm{L} \mathrm{L}^{-1}$ & This study \\
\hline
\end{tabular}




\section{Conclusions}

A 3D-GA prepared by chemical reduction of an aqueous solution of graphene oxides followed by freeze-drying treatment was packed in an SPE cartridge for the extraction of OPPs. Because of its well-developed porous structure and large specific surface area, 3D-GA was evinced to be a very efficient adsorbent for the SPE enrichment and purification of OPPs.

Coupled with GC/MS techniques, the 3D-GA packed SPE was applied to the determination of six OPPs (trichlorfon, ethoprophos, dimethoate, fenitrothion, parathion and fenthion) in water samples, and satisfactory linearity, high precision, good repeatability and high recovery were achieved. The results presented herein indicate that the present method could be used efficiently for the determination of trace OPPs in a variety of water samples.

\section{Conflicts of interest}

There are no conflicts to declare.

\section{Acknowledgements}

This research was supported by the National Natural Science Foundation of China (Grants 51572071, 51072047) and the Program for Innovative Research Team in University (The Ministry of Education of China, Grant IRT-1237).

\section{References}

1 J. Hassan and M. Sarkouhi, Arabian J. Chem., 2016, 9, 38.

2 H. R. Nodeh, W. A. W. Ibrahim, M. A. Kamboh and M. M. Sanagi, Chemosphere, 2017, 166, 21.

3 Z. M. Wang, X. Zhao, X. Xu, L. J. Wu, R. Su, Y. J. Zhao, C. F. Jiang, H. Q. Zhang, Q. Ma, C. M. Lu and D. M. Dong, Anal. Chim. Acta, 2013, 760, 60.

4 G. Cinelli, P. Avino, I. Notardonato and M. V. Russo, Anal. Methods, 2014, 6, 782.

5 S. Boulanouar, S. Mezzache, A. Combès and V. Pichon, Talanta, 2017, 176, 465.

6 P. Zohrabi, M. Shamsipur, M. Hash and B. Hashemi, Talanta, 2016, 160, 340.

7 L. J. Wu, Y. Song, M. Z. Hu, X. Xu, H. Q. Zhang, A. M. Yu and Z. M. Wang, Talanta, 2015, 134, 366.
8 R. Su, X. Xu, X. H. Wang, D. Li, X. Y. Li, H. Q. Zhang and A. M. Yu, J. Chromatogr. B: Anal. Technol. Biomed. Life Sci., 2011, 879, 3423.

9 V. Sharifi, A. Abbasi and A. Nosrati, J. Food Drug Anal., 2016, 24, 264.

10 L. J. Wu, M. Z. Hu, Z. C. Li, Y. Song, H. Q. Zhang, A. M. Yu, Q. Ma and Z. M. Wang, J. Chromatogr. A, 2015, 1407, 42.

11 L. Jiang, T. J. Huang, S. Feng and J. D. Wang, J. Chromatogr. A, 2016, 1456, 49.

12 R. Su, D. Li, X. H. Wang, H. M. Yang, X. Y. Shi and S. Y. Liu, J. Chromatogr. B: Anal. Technol. Biomed. Life Sci., 2016, 1022, 141.

13 H. B. Zheng, Q. Zhao, J. Z. Mo, Y. Q. Huang, Y. B. Luo, Q. W. Yu and Y. Q. Feng, J. Chromatogr. A, 2013, 1300, 127.

14 I. Notardonato, P. Avino, G. Cinelli and M. V. Russo, RSC Adv., 2014, 4, 42424.

15 M. H. M. Facure, L. A. Mercante, L. H. C. Mattoso and D. S. Correa, Talanta, 2017, 167, 59.

16 C. Y. Liu, H. Y. Liu, A. R. Xu, K. Y. Tang, Y. Huang and C. Lu, J. Alloys Compd., 2017, 714, 522.

17 Y. C. Shi, A. J. Wang, X. L. Wu, J. R. Chen and J. J. Feng, J. Colloid Interface Sci., 2016, 484, 254.

18 B. Tan, H. M. Zhao, Y. B. Zhang, X. Quan, Z. H. He, W. T. Zheng and B. Y. Shi, J. Colloid Interface Sci., 2018, 512, 853.

19 S. Mahpishanian and H. Sereshti, J. Chromatogr. A, 2016, 1443, 43.

20 X. M. Wang, M. X. Lu, H. Wang, Y. F. Pei, H. H. Rao and X. Z. Du, Sep. Purif. Technol., 2015, 153, 7.

21 X. M. Wang, M. X. Lu, H. Wang, P. F. Huang, X. M. Ma, C. Cao and X. Z. Du, New J. Chem., 2016, 40, 6308.

22 W. S. Hummers and R. E. Offeman, J. Am. Chem. Soc., 1958, 80, 1339.

23 Q. Liu, J. B. Shi, L. X. Zeng, T. Wang, Y. Y. Cai and G. B. Jiang, J. Chromatogr. A, 2011, 1218, 197.

24 I. Kondratowicz, K. Żelechowska, M. Nadolska, A. Jażdżewska and M. Gazda, Colloids Surf., A, 2017, 528, 65. 25 S. Z. Zu and B. H. Han, J. Phys. Chem. C, 2009, 113, 13651.

26 M. A. Farajzadeh, M. R. A. Mogaddam, S. R. Aghdam, N. Nouri and M. Bamorrowat, Food Chem., 2016, 212, 198.

27 X. S. Zhao, W. J. Kong, J. H. Wei and M. H. Yang, Food Chem., 2014, 162, 270.

28 Z. P. Cheng, F. S. Dong, J. Xu, X. G. Liu, X. H. Wu, Z. L. Chen, X. L. Pan, J. Gan and Y. Q. Zheng, Food Chem., 2017, 231, 365. 